\title{
Nuclear Pore Complex
}

National Cancer Institute

\section{Source}

National Cancer Institute. Nuclear Pore Complex. NCI Thesaurus. Code C13951.

Signal-mediated nuclear import and export proceed through the nuclear pore complex

(NPC). NPC is comprised of approximately 50 unique proteins collectively known as

nucleoporins. NPC functions in the nuclear transport of protein and RNA. (NCI) 